\title{
Grain growth from homogeneous initial conditions: Anomalous grain growth and special scaling states
}

\author{
Yi Jiang, ${ }^{*}$ José C. M. Mombach, ${ }^{\dagger}$ and James A. Glazier \\ Department of Physics, University of Notre Dame, Notre Dame, Indiana 46556
}

(Received 19 May 1995)

\begin{abstract}
We used the large- $Q$ Potts model on a two-dimensional lattice to study the evolution of the disordered cluster developed from a perfect hexagonal lattice with a single defect. The distribution functions were not stable, while the average area and the number of grains in the cluster grew linearly in time. However, the grains at the boundary of the cluster formed a well defined region which reached a special scaling state with time invariant distributions but no scale change, contrary to the result of Levitan [Boris Levitan, Phys. Rev. Lett. 72, 4057 (1994)]. The rate of propagation of disorder is the same as the rate of growth of the cluster. Abnormal grain growth can occur without anisotropy of surface energy. It requires only widely spaced, modest size differences in an initially homogeneous array of grains.
\end{abstract}

PACS number(s): 82.70.Rr, 02.50.-r, 05.70.Ln, 81.35.+k

Two-dimensional coarsening occurs in a wide range of materials. In spite of a few complications, the evolution of grains is governed by the simple and well understood von Neumann's law:

$$
\frac{d a_{n}}{d t}=\kappa(n-6)
$$

where $\kappa$ is a system-dependent diffusion constant, i.e., the rate of change of area of a grain $a_{n}$ depends only on its number of sides $n$. Therefore grains with more than six sides expand, while grains with fewer than six sides shrink and eventually disappear. When a grain disappears, its neighboring grains may gain or lose sides. In such processes, side redistributions occur that determine the topological evolution of the pattern. In particular, experiments have revealed that the pattern evolves into a scaling state in which the side distribution functions $\rho(n)$, and the rescaled size distribution functions $\rho(a /\langle a\rangle)$, where $\langle a\rangle$ is the mean area, remain constant, while the length scale increases with time [1,2]. This scaling state is universal, since all reasonably homogeneous initial conditions evolve to it. With both von Neumann's law and the scaling state assumption, the average area of grains grows linearly with time [3].

Nevertheless, in many materials coarsening does not reach a scaling state, e.g., in abnormal grain growth. Abnormal grain growth in metals is believed to occur when surface energy depends on relative grain orientation, when lattice anisotropy is strong [4]. At the free surface of a thin film, normal grain growth stagnates due to the pinning of grain boundaries by surface grooving. Beyond this stage, a few (abnormal) grains grow by consuming their neighbors. The usual explanation is that anisotropic free surface energy varies locally [5].

\footnotetext{
${ }^{*}$ Corresponding author. Electronic address: yjiang@lully.phys.nd.edu

${ }^{\dagger}$ Permanent address: Instituto de Física, Universidade Federal do Rio Grande do Sul, Caixa Postal 15051, 91501-970 Porto Alegre, RS, Brazil. Electronic address: jose@if1.if.ufrgs.br
}

In a recent study of time evolution of two-dimensional soap froth with a single defect [6], Levitan challenges the common wisdom that the scaling state dynamics does not depend on the initial condition. Using a mean-field treatment, Levitan claims that the long-time distribution function $\rho(n)$ is different for generic initial conditions or for an initial hexagonal lattice with only one defect. He also found the scaling law $n_{c}(t) \sim t$ [7], where $n_{c}$ is the number of grains in the cluster. In a comment, Weaire restates the evidence for scaling in experiment and draws attention to the importance of $\mu_{2}$, the second moment of sides distribution, which is not mentioned in Levitan's work [8]. Sire concentrates on the discrepancy between the topological model and the numerical result from simulations of the area model [9]. In order to account for the discrepancy, Sire introduces a model that gives a scaling law $n_{c}(t) \sim t \ln \left(t^{2}\right)$. Unfortunately in this model, Sire assumes that grains in the disordered cluster are in the usual scaling state. This assumption is neither trivial nor obvious. Both comments address the convergence of the convolution. However, we think this question cannot be answered by any mean-field theory, since the final state consists of distinct classes with fixed spatial relations.

We evolve a special initial condition: a single big grain (area greater than the mean area of the hexagons) in a perfect regular hexagonal lattice. Without a defect, the lattice would be stable for all time. The defect functions as a seed for the evolution. Therefore the froth consists of two parts: the evolving neighborhood of the defect, and the rest of the lattice which does not evolve. As the froth evolves in time, the boundary of the disordered region propagates outwards. We would like to find out whether the coarsening reaches a scaling state, its topological distribution, and its growth rate.

We use a large- $Q$ Potts model [10] in our simulation, with the Potts Hamiltonian:

$$
\mathscr{H}=\sum_{(i, j)} \sum_{\left(i^{\prime}, j^{\prime}\right)}\left[\delta_{\sigma_{i, j}, \sigma_{i^{\prime}, j^{\prime}}}-1\right],
$$

where $\sigma_{i, j}$ is the spin value at lattice site $(i, j)$ ranging from 1 to $Q(Q>10000)$ and $\left(i^{\prime}, j^{\prime}\right)$ is the neighbor of $(i, j)$. The range of the second sum affects the nature of the interaction. The spins evolve by the Monte Carlo procedure, where a 
spin is chosen at random, and flipped only if the flip lowers the total pattern energy. This algorithm corresponds to the zero temperature limit. We run on a $2000 \times 2000$ lattice until the disordered cluster reaches the boundaries of the lattice. For our simulation, we choose a fourth nearest neighbor square lattice (lattice anisotropy $\eta_{c}=1.037$ ), which is known to evolve in a manner very close to ideal grain growth [11].

In agreement with Levitan [6], we define the disordered cluster to consist of all grains with at least one nonhexagonal neighbor, yielding a large grain in the center and a boundary of grains around it. Figure 1 shows snapshots of the evolution. The time unit used is the Monte Carlo step (MCS), defined as $N_{0}$ attempts to flip the spins, where $N_{0}$ $\left(4 \times 10^{6}\right)$ is the number of lattice spin sites in the pattern. As we can see from the figures, the center grain grows much faster than the grains at its boundary, whose average area randomly fluctuates around a value $\left\langle a^{\prime}\right\rangle=(0.88 \pm 0.08) a_{0}$, where $a_{0}$ is the initial area of the hexagons. The grains outside the cluster remain unchanged. The disordered cluster maintains a bilayer of grains around the large grain; that is, the large grain grows at the same rate as disorder propagates outward in the pattern. The diameter of the cluster grows linearly in time, while the area of the cluster grows quadratically. This result is trivial if we consider a big grain growing without affecting neighboring unevolving small grains. Its number of sides (number of neighbors) is proportional to its perimeter, i.e., $n \sim d$, with $d$ being the diameter of the big grain, so the normal velocity of the propagating front is constant. Using von Neumann's law, we have

$$
\frac{d a_{n}}{d t} \sim n \sim d \sim \sqrt{a_{n}} .
$$

Equation (3) gives $a \sim t^{2}$ and $d \sim t$. Also, the diameter and the total area of the cluster are chiefly determined by the large center grain. Therefore we observe similar behavior for the growth of the cluster (Fig. 2).

The number of the grains in the cluster grows linearly (Fig. 2). This result is again trivial, although not obvious, since the total number of grains in the cluster $n_{c} \sim n$ (the number of neighbors of the center grain), $n_{c} \sim t$, the same as in Levitan's simulation. The average area of the grains in the cluster, as we predict $\left(\langle a\rangle=\langle a / n\rangle \sim t^{2} / t \sim t\right)$, grows linearly, accidentally mimicking a normal scaling state, whereas the average area of the grains in the cluster boundary is constant.

The tail of the topological distribution function, $\rho(n)$, extends towards larger and larger values of $n$, corresponding to the large center grain. The peak stays at $n=6$ due to our definition of the cluster. The second moment of the topological distribution, dominated by the large grain, grows linearly [Fig. 3(b)], consistent with the data of Aboav [12], for the transient behavior of samples which are initially almost hexagonal. If we exclude the large grain in the center, the rest of the cluster presents a $\mu_{2}^{\prime}$ fluctuating around a constant value of about 0.7 [Fig. 3(b)] and the topological distribution is constant [Fig. 3(a)]. Thus, self-similarity is preserved in the boundary of the cluster, yielding a special scaling state, though not in the way claimed by Levitan. In neither case do we ever reach a normal scaling state. The mean-field theory fails because in this pattern, the large grain, the boundary grains, and the remaining hexagonal grains form three dis-
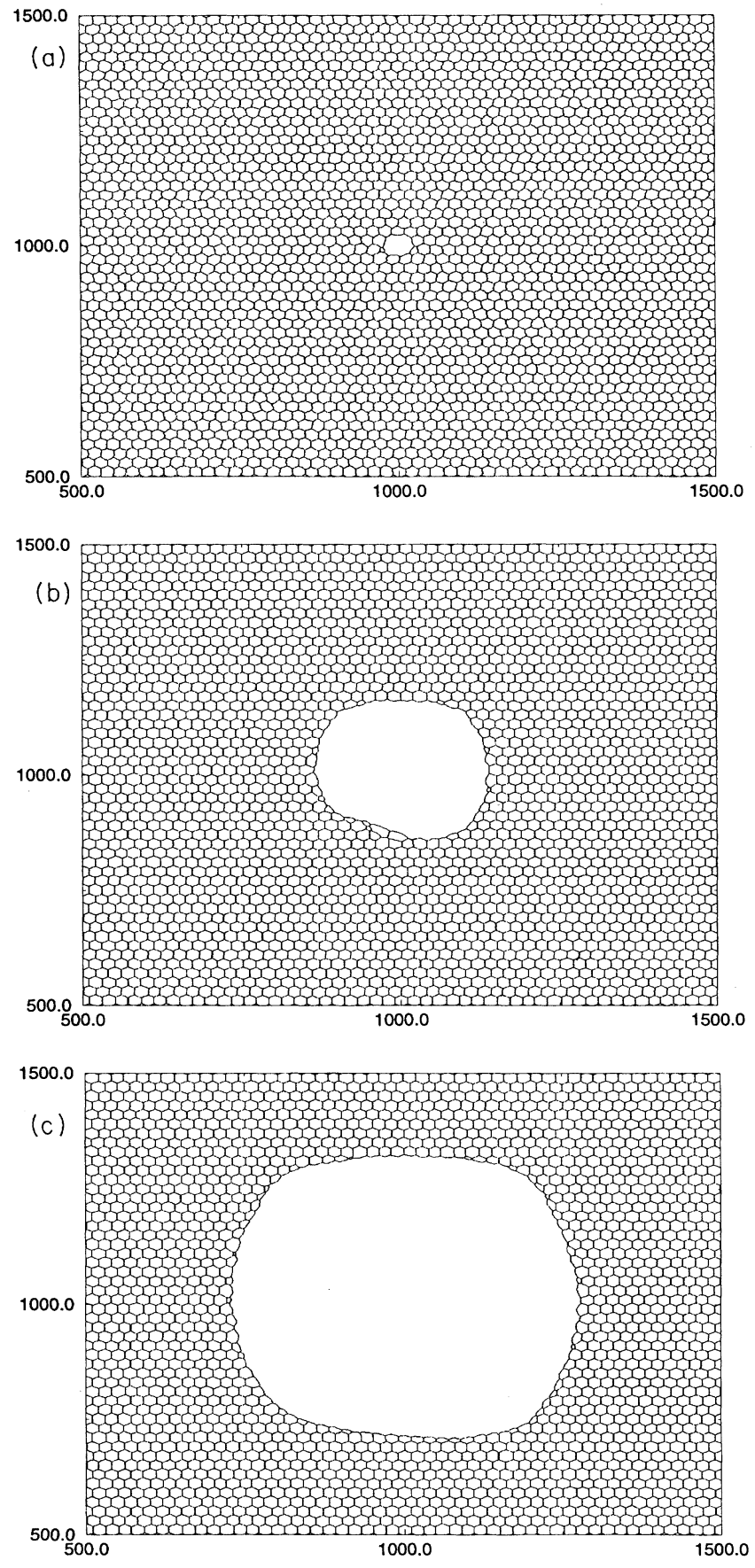

FIG. 1. Snapshots of time evolution of an initially hexagonal pattern with a single defect: (a) $0 \mathrm{MCS}$, (b) $5000 \mathrm{MCS}$, (c) 10000 MCS.

tinct classes with fixed spatial relations: exactly. opposite to the homogeneous mean-field assumption.

Theoretical work has attempted to show that the scaling state is reached for all initial conditions at long times, and to derive the distribution functions. Since no exact solutions are available for physically relevant dynamics, mean-field approximations were used. Some of these studies revealed the existence of an entire family of stable scaling solutions, rais- 


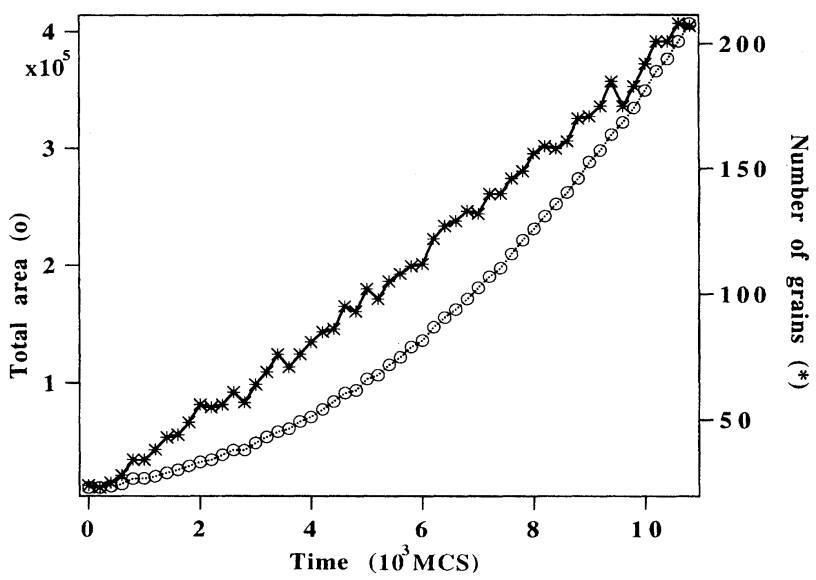

FIG. 2. Evolution of the disordered cluster: Total area of the cluster $(O)$ grows quadratically and the number of grains in the cluster $(*)$ grows linearly.

ing some intriguing questions about the mechanism by which one particular solution is universally selected [13-17].

The transition time to a scaling state strongly depends on the randomness of the initial conditions [18]. To quickly reach the scaling regime in computer simulations requires a sufficiently random initial lattice of grains. If we begin with a very regular array with a few widely spaced defects, we would thus see the rapid growth of a few grains, as in abnormal grain growth.

In the evolution of a single defect in an isotropic hexagonal lattice, the large grain (from the defect) grows at the same rate as disorder propagates outward. The statistics of the disordered cluster shows that the topological distribution diverges in time, while the average area of the grains in the cluster grows linearly, a result supported by Aboav's data and previous simulations. However, excluding the large center grain, side and area distributions are steady for the boundary of the cluster. Thus, at the boundary of the large grain, we find a special scaling state, composed of different grains at different times. It has a constant average area $\left\langle a^{\prime}\right\rangle$ $=(0.88 \pm 0.08) a_{0}$, and nontrivial distributions with $\mu_{2}^{\prime}=0.71 \pm 0.17$, i.e., time invariant statistics but no scale change. The mean-field theory of Levitan fails. The result
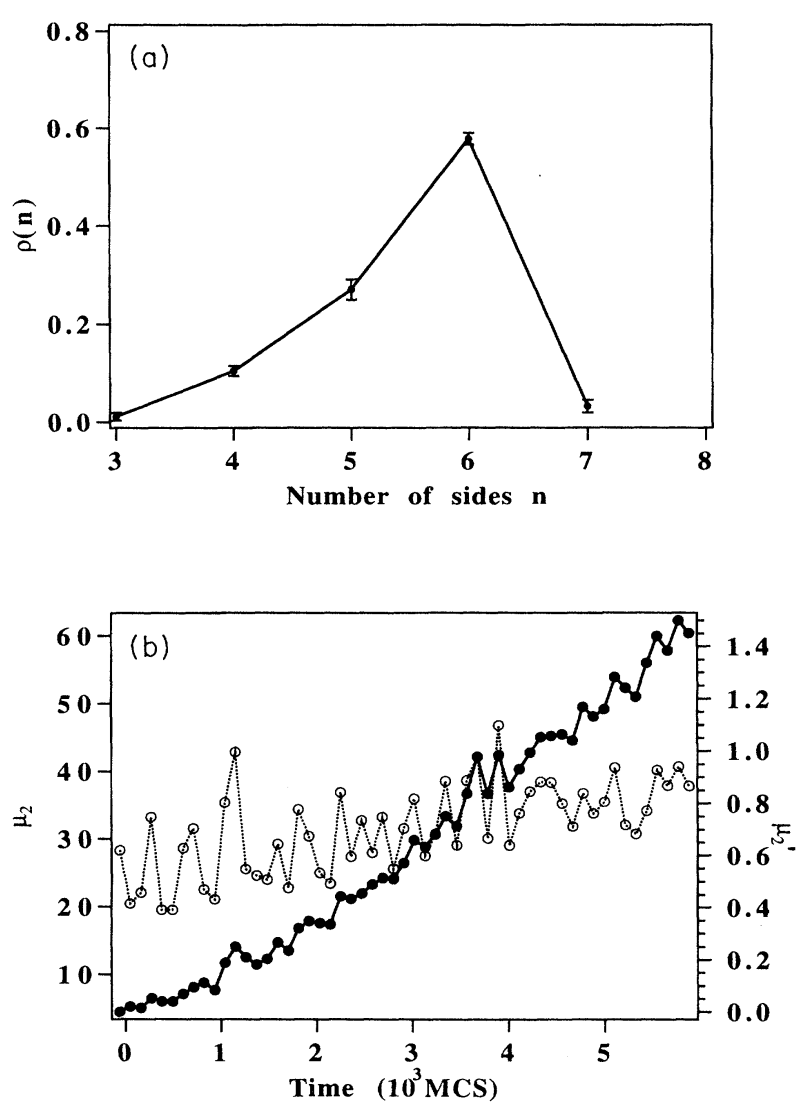

FIG. 3. Topological distributions: (a) Topological distribution, $\rho(n)$, excluding the large center grain, averaged over six different times between $6000 \mathrm{MCS}$ and $10600 \mathrm{MCS}$. Error bars are standard deviation of the mean. (b) The second moment of $\rho(n)$ of the cluster $\left(\mu_{2}\right.$, bullets) and the boundary $\left(\mu_{2}^{\prime}\right.$, circles).

also shows that abnormal grain growth could occur in materials provided their crystallites are highly uniform and ordered, without any anisotropy or fluctuations of surface energy.

The authors acknowledge support from Ford Motor Company, Exxon Educational Foundation, ACS/PRF and NSF/ NYI DMR-9257011.
[1] J. Stavans and J. A. Glazier, Phys. Rev. Lett. 62, 1318 (1989); J. A. Glazier, S. P. Gross, and J. Stavans, Phys. Rev. A 36, 306 (1987).

[2] J. A. Glazier, Ph.D. thesis, University of Chicago, 1989 (unpublished).

[3] W. W. Mullins, Scr. Metall. 22, 1441 (1988).

[4] A. D. Rollett, D. J. Srolovitz, and M. P. Anderson, Acta Metall. 37, 1227 (1989); G. S. Grest, M. P. Anderson, D. J. Srolovitz, and A. D. Rollett, Scr. Metall. 24, 661 (1990).

[5] H. J. Frost, C. V. Thompson, and D. T. Walton, Mater. Sci. Forum 94, 2 (1992); Acta Metall. 38, 1455 (1990); 40, 779 (1992).

[6] Boris Levitan, Phys. Rev. Lett. 72, 4057 (1994).
[7] Levitan found $n_{c} \sim \tau^{0.5}$, where time $\tau$ was defined as the number of grains annihilated. Converting to the realistic time $t \sim \tau^{0.5}$ yields $n_{c} \sim t$.

[8] D. Weaire, Phys. Rev. Lett. 74, 3710 (1995).

[9] C. Sire, Phys. Rev. Lett. 74, 3708 (1995).

[10] M. P. Anderson, D. J. Srolovitz, G. S. Grest, and P. S. Sahni, Acta Metall. 32, 783 (1984); J. A. Glazier, M. P. Anderson, and G. S. Grest, Philos. Mag. B 62, 615 (1990).

[11] E. Holm, J. A. Glazier, D. J. Srolovitz, and G. S. Grest, Phys. Rev. A 43, 2262 (1991).

[12] D. A. Aboav, Metallography 13, 43 (1980).

[13] J. Stavans, E. Domany, and D. Mukamel, Europhys. Lett. 15, 419 (1991). 
[14] D. Weaire and H. Lei, Philos. Mag. Lett. 62, 427 (1990).

[15] D. Segel, D. Mukamel, O. Krichevsky, and J. Stavans, Phys. Rev. E 47, 812 (1993).

[16] M. Marder, Phys. Rev. A 36, 438 (1993).
[17] H. Flyvbjerg, Phys. Rev. E 47, 4037 (1993).

[18] J. A. Glazier and D. Weaire, J. Phys. Condensed Matter 4, 1867 (1992) 